\title{
Cholera in Haiti: Redefining Emergency Public Health Philosophy
}

\author{
Samuel J. Stratton, MD, MPH
}

The 2010 earthquake disaster in Haiti has been studied from many perspectives. Scientific evaluation of the Haiti disaster has helped refine disaster health response and emergency public health management. For example, the variation among humanitarian response teams in surgical aggressiveness for amputation of injured limbs of earthquake victims has been controversial. ${ }^{1,2}$ Further, the limitations in medical follow-up, rehabilitation, and psychologicalsocial counseling for those who underwent amputation have been a challenge that was not anticipated or fully addressed. ${ }^{2,3}$ A chaotic as opposed to an organized humanitarian disaster medical response seems to extend the potential for human suffering well beyond that expected for the immediate event.

More subtle than the effects of emergency surgical procedures and other emergency humanitarian response actions has been the re-introduction of cholera into the Haiti environment. During the earthquake response period, there was little worry that cholera would be a factor. The disease had been eradicated in Haiti more than a century earlier. Yet the outbreak of cholera soon after the earthquake did occur, contradicting expert public health "knowledge." None in the public health profession expected a disease outbreak from a pathogen that was not endemic to the region of the disaster.

Nine months after the earthquake struck Haiti, the Haitian Ministry of Public Health and Population reported the cholera outbreak. The outbreak, which became an epidemic, was another disaster for the people of Haiti. In the two years after the initial October 2010 reports of cholera in Haiti, 604,634 cases of infection were reported, with 329,697 hospitalizations and 7,436 deaths directly attributed to the infection. ${ }^{4}$ The epidemic cholera strain was found to be $V$. cholera O1, a strain that was not endemic in the western hemisphere but rather found in southern Asia and eastern Africa. ${ }^{5}$ The introduction of a virulent strain of cholera into an immunologically naïve population resulted in rapid transmission of infection with high morbidity and mortality. The epidemic further crippled a population already severely damaged by the January 2010 earthquake.

The Haiti cholera epidemic has become one of the most studied in history. Best evidence indicates that the cholera strain was introduced into Haiti from human fecal contamination of a local stream that drained into the Artibonite River, with spread to populated areas downstream in the river valley. ${ }^{6}$ During the initial stages of the epidemic, there were multiple introductions of the disease into Haitian refugee camps which housed persons particularly vulnerable to infection. The disease further spread into the Dominican Republic and into Florida (USA) without secondary spread from those sites due to an intact infrastructure for water and sewage treatment and management.

\section{References}

1. Chackungal S, Nickerson JW, Knowlton LM, et al. Best practice guidelines on surgical response in disasters and humanitarian emergencies: report of the 2011 Humanitarian Action Summit Working Group on Surgical Issues within the Humanitarian Space. Prehosp Disaster Med. 2011;26(6):429-437.
While controversial, epidemiologic evidence associates the cholera contamination of the Haiti water supply with a visiting international peacekeeping force that responded to the earthquake event. The peacekeeping force arrived from southern Asia where the strain of cholera that was introduced into Haiti is endemic.

Prior to the earthquake, Haiti had undergone long-term degradation of its environmental health infrastructure, including water and sanitation resources. With the earthquake event, safe water and sanitation was further challenged with infrastructure destruction and mass movement of earthquake victims. This made the region vulnerable to environmental challenges. But, none who accepted the emergency public health beliefs at the time of the earthquake would have anticipated an epidemic caused by a nonendemic infectious agent. Also of importance was the spread of cholera from the epidemic region to the Dominican Republic and regions in the US. It appears that international travelers who were likely going to the aid of disaster-stricken populations became carriers of the infection to other world locations where cholera is not endemic.

The Haiti cholera epidemic redefines emergency public health philosophy. No longer can it be assumed that during a disaster event, the only epidemic to expect is one that is related to endemic infectious agents. Rather, in addition to endemic agents, an epidemic related to agents imported by the response teams and efforts must be anticipated. In addition, expect that an epidemic that is the result of the disaster can be further spread by international travelers who visit the disaster region.

Prioritizing sanitation during disaster planning and response is another important lesson from the Haiti cholera epidemic. Damage to water and sanitation infrastructure is an important consideration when assessing the vulnerability of a disasteraffected population. Environmental public health efforts appear to be as important to a population as other public health efforts. Further, protecting a disaster-affected population from imported disease is crucial, and use of appropriate and effective sanitation practices by responders should be emphasized. Proper vaccination and chemoprophylaxis of responders is also important to limit infection and spread of disease both within the disaster zone and for potential transmission out of the disaster area.

The Haiti cholera epidemic redefines emergency public health philosophy. No longer can it be assumed that only endemic diseases should be expected in a disaster zone; rather, expect that disease can be imported and exported along with the response efforts. In addition, sanitation using the most effective methods should be a priority of disaster-responding groups and individuals.

2. Redmond $\mathrm{AD}$, Mardel S, Taithe $\mathrm{B}$, et al. A qualitative and quantitative study of the surgical and rehabilitation response to the earthquake in Haiti, January 2010. Prehosp Disaster Med. 2011;26(6):449-456. 
3. Patel PP, Russell J, Allden K, et al. Transitioning mental health and psychosocial support: from short-term emergency to sustainable post-disaster development. Humanitarian Action Summit 2011. Prehosp Disaster Med. 2011; 26(6):470-481.

4. Barzilay EJ, Schaad N, Magloire R, et al. Cholera surveillance during the Haiti epidemic - the first 2 years. NEJM. 2013;368(7):599-609.
5. Piarroux R, Barrals R, Faucher B, et al. Understanding the cholera epidemic, Haiti. Emerg Infect Dis. 2011;17(7):1161-1167.

6. Dowell SF, Braden CR. Implications of the introduction of cholera to Haiti. Emerg Infect Dis. 2011;17(7):1299-1300.

doi:10.1017/S1049023X13000320 